\title{
An estimation of the plasma parameters in the solar corona using quasi-periodic metric type III radio burst emission
}

\author{
R. Ramesh ${ }^{1}$, A. Satya Narayanan ${ }^{2}$, C. Kathiravan ${ }^{2}$, Ch. V. Sastry ${ }^{3}$, and N. Udaya Shankar ${ }^{4}$ \\ ${ }^{1}$ Centre for Research and Education in Science \& Technology, Indian Institute of Astrophysics, Hosakote 562 114, India \\ e-mail: ramesh@iiap.res.in \\ 2 Indian Institute of Astrophysics, Bangalore 560 034, India \\ 3 “MEENAKSHI", No. 3, 2nd Cross, 1st Block, Koramangala, Bangalore 560 034, India \\ ${ }^{4}$ Raman Research Institute, Bangalore 560 080, India
}

Received 21 April 2004 / Accepted 19 October 2004

\begin{abstract}
We report radio observations of quasi-periodic type III burst emission from the solar corona in close association with the 2B/X2.8 flare of December 11, 2001 at two frequencies (151.5 and $109 \mathrm{MHz}$ ). The measured mean period was $\approx 11.6 \mathrm{~s}$. We attempted to infer the plasma parameters of the region where the electron beams responsible for the observed type III emission were accelerated/injected using our data, and the values are the following: the Alfvén speed $\left(v_{\mathrm{A}}\right)=873 \pm 427 \mathrm{~km} \mathrm{~s}^{-1}$; the electron density $\left(N_{\mathrm{e}}\right) \approx 3.09 \times 10^{9} \mathrm{~cm}^{-3}$; the magnetic field strength $(B)=22 \pm 11 \mathrm{G}$.
\end{abstract}

Key words. Sun: activity - Sun: flares - Sun: oscillations - Sun: radio radiation

\section{Introduction}

Type III solar radio bursts, characterized by a rapid frequency drift from high to low frequencies (at rates of $\sim 100 \mathrm{MHz} \mathrm{s}^{-1}$ ) and lifetime of a few seconds were observed for the first time by Wild (1950). They are considered to be radio wave signature of electron beams propagating through the corona and interplanetary medium (see Lin 1974 for a review). The bursts are common whenever a moderately bright active region is on the visible hemisphere of the Sun. Most bursts occur sporadically during periods of no activity, although Zirin \& Lazareff (1975) consider that almost all metric type III bursts are accompanied by small, weak chromospheric brightening. Groups of type III bursts lasting for about $5 \mathrm{~min}$ are generally associated with flares (seen in X-rays and/or $\mathrm{H} \alpha$ ). They are a classic signature of the impulsive phase of flares in which the radiation is primarily non-thermal. It is believed that each individual burst is radiation from electron streams moving outwards through the corona and exciting plasma oscillations of progressively diminishing frequency as the stream passes through the coronal plasma of decreasing electron density. The occurence of non-thermal bursts is a clear indication of particle acceleration during an event. Type III bursts thus trace the path of an electron beam from near the acceleration site. In view of the above, a study of the temporal behaviour of type III radio bursts is now considered to be a simple and useful tool to investigate the time structure of particle acceleration in solar flares (see Aschwanden 2002, and the references therein). Also note that the generation of metric radio emission by non-thermal electron beams is very efficient due to the coherent nature of the emission mechanism. In studies on the quasi-periodicity of type III radio bursts, the observed period is usually attributed to the time scale on which an Alfvénic disturbance traverses the accelerator source (Mangeney \& Pick 1989; Zhao et al. 1991). In this situation, we present radio observations of quasi-periodic type III radio burst emission from the solar corona at two different frequencies (151.5 and $109 \mathrm{MHz})$ and derive the plasma parameters of the electron acceleration/injection region assuming that the periodicity is due to a modulation of the electron acceleration/injection process responsible for the observed type III emission by oscillations setup in/near the former by a propagating MHD disturbance generated in the aftermath of the associated $\mathrm{H} \alpha$ flare.

\section{Observations}

The radio data reported were obtained on December 11, 2001 with the Gauribidanur radioheliograph (GRH, Ramesh et al. 1998) operating near Bangalore in India, and the E-W arm of the Mauritius radio telescope (MRT, Golap et al. 1998). The observing frequencies of the two instruments are 109 and 151.5 MHz, respectively. Figure 1 shows the temporal evolution of the radio emission from the Sun observed with the MRT and GRH during the interval 08:01-08:06 UT on December 11, 2001. The integration time used for the MRT observations (indicated by solid line) was $1 \mathrm{~s}$. One can notice two well-defined phases of quasi-periodic emission: (1) 08:02:23-08:03:48 UT; and (2) 08:04:00-08:05:45 UT, in the latter. We estimated 


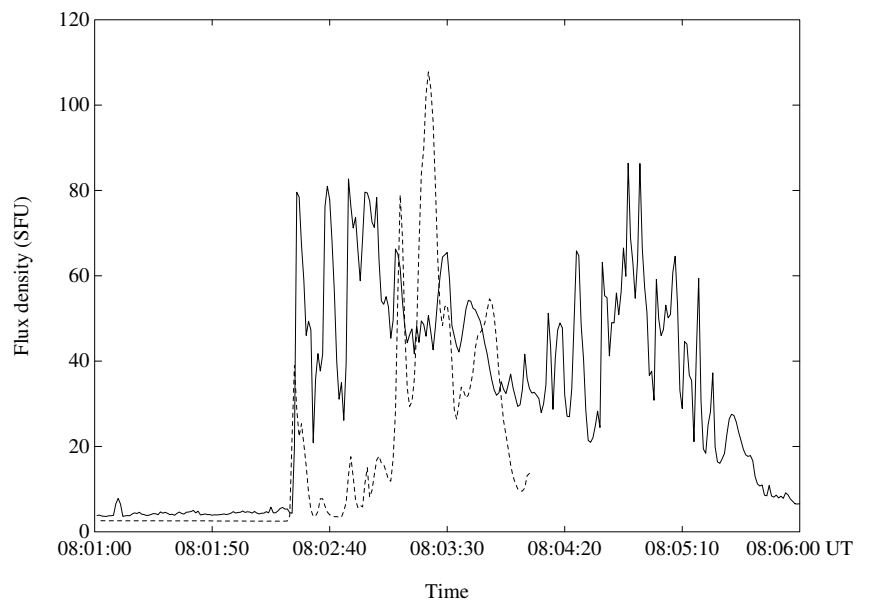

Fig. 1. Time profile of the radio burst emission from Sun observed with the GRH at $109 \mathrm{MHz}$ (indicated by dotted line) and MRT at $151.5 \mathrm{MHz}$ (indicated by solid line) on December 11, 2001 during the period 08:01:00-08:06:00 UT. The transient emission in the interval 08:02:23-08:04:00 UT (both the GRH and MRT data) correspond to a type III burst group. The discontinuity in the GRH data after 08:04:03 UT is due to the lack of observations beyond that time on that day. The 2nd phase of the MRT data, i.e. in the interval 08:04:00-08:05:45 UT correspond to a type V burst.

their period from the time interval between the adjacent bursts and the average values are 11.9 and $7.7 \mathrm{~s}$, respectively. The corresponding peak flux density values are 80 and 85 SFU $\left(\mathrm{SFU}=\right.$ solar flux unit $\left.=10^{-22} \mathrm{~W} / \mathrm{m}^{2} / \mathrm{Hz}\right)$. The dashed line indicates GRH observations on the same day, with an integration time of $256 \mathrm{~ms}$. One can notice clear quasi-periodicity here also in the time range 08:02:23-08:04:00 UT. This correlates well with the first phase of MRT observations. The mean period was estimated in the same manner as above, and the value is $\approx 11.4 \mathrm{~s}$. The peak flux density is $110 \mathrm{SFU}$. We would like to point here that no data was available after 08:04:03 UT at $109 \mathrm{MHz}$ due to the lack of GRH observations beyond that time on that day. One can notice that the average period at both 151.5 and $109 \mathrm{MHz}$ are almost the same. The above radio events were closely associated with a $2 \mathrm{~B}$ class $\mathrm{H} \alpha$ flare from AR 9733 (N16E41) in the interval 08:02-09:03 UT, with peak at 08:05 UT. There was also a GOES X2.8 class X-ray flare around the same time, i.e. 07:58-08:14, with peak at 08:08 UT (Solar Geophysical Data, June 2002). Figure 2 shows the half-power contours of the radioheliogram obtained with the GRH at 08:02:30, 08:03:00 and 08:03:30 UT. There is a close correspondence between the location of the discrete regions of intense radio emission and the flare site mentioned above. Figure 3 shows the dynamic spectrum of the radio emission from the solar corona obtained with the IZMIRAN radiospectrograph on December 11, 2001 in the time interval 08:01:00-08:06:00 UT. One can notice parallel drifting bands of burst emission starting from 08:02:30 UT and lasting for about a minute. The above event was classified as a type III group (Solar Geophysical Data, February 2002). A comparison of the spectral data and the single frequency records indicate that the MRT (first phase) and GRH time profile in Fig. 1

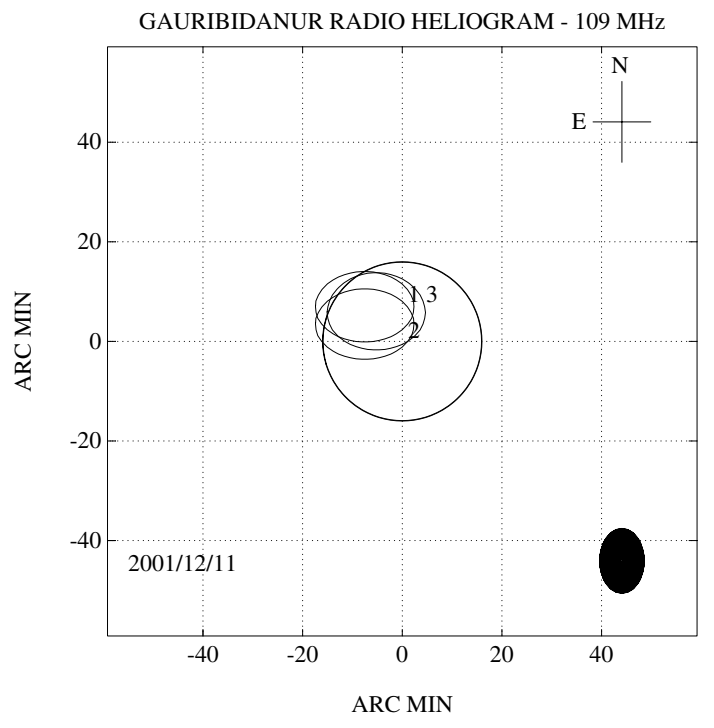

Fig. 2. Half-power contours of the radioheliogram obtained with the GRH on December 11, 2001 at 08:02:30 (1); 08:03:00 (2); and 08:03:30 UT (3), the various stages of the observed quasi-periodic radio emission. One can notice that there is a good correspondence between the centroid of the radio sources and the flare site mentioned in the text. The estimated peak radio brightness temperature is $\approx 8.13 \times 10^{8} \mathrm{~K}$ and it corresponds to the image obtained at 08:03:00 UT. The open circle at the center is the solar limb. The size of the GRH beam at $109 \mathrm{MHz}$ is indicated at the lower right corner.

corresponds to the aforementioned burst. The scatter in the centroid of the radio sources in Fig. 2 also points to an association with a type III group, since the latter are considered to escape along large scale diverging magnetic field lines connected to a common acceleration/injection site (Mercier 1975; Pick \& Ji 1986). The second part of MRT observations in Fig. 1 is probably related to the intense emission following the type III group in Fig. 3. It is most likely a type V burst which generally appears as an extension to a small percentage of type III bursts (Wild et al. 1959). The published reports in the Solar Geophysical Data (February 2002) also indicate that it is a type $\mathrm{V}$ burst. A detailed analysis of this event will be reported separately. There was also a coronal type II burst following the type III/V events, in the frequency range $\approx 192-46 \mathrm{MHz}$. It started at 08:08 UT (192 MHz) and was first noticed at $46 \mathrm{MHz}$ around 08:13:30 UT (Fig. 4). Assuming a 10× BaumbachAllen model (Baumbach 1937; Allen 1947) for the density distribution in the active region corona, we calculated the speed of the associated MHD shock from the observed drift rate of the burst $\left(\sim 0.4 \mathrm{MHz} \mathrm{s}^{-1}\right)$ and the value is about $991 \mathrm{~km} \mathrm{~s}^{-1}$.

\section{Analysis and results}

It is now well known that metric radio observations of solar type III burst group sometimes show quasi-periodic fluctuations (Wild et al. 1963; Janssens et al. 1973; Mangeney \& Pick 1989; Aschwanden et al. 1994; Ramesh et al. 2003). They consist of several widely separated elementary components which radiate quasi-simultaneously or successively (Vlahos et al. 1986, and the references therein). One of the suggested 


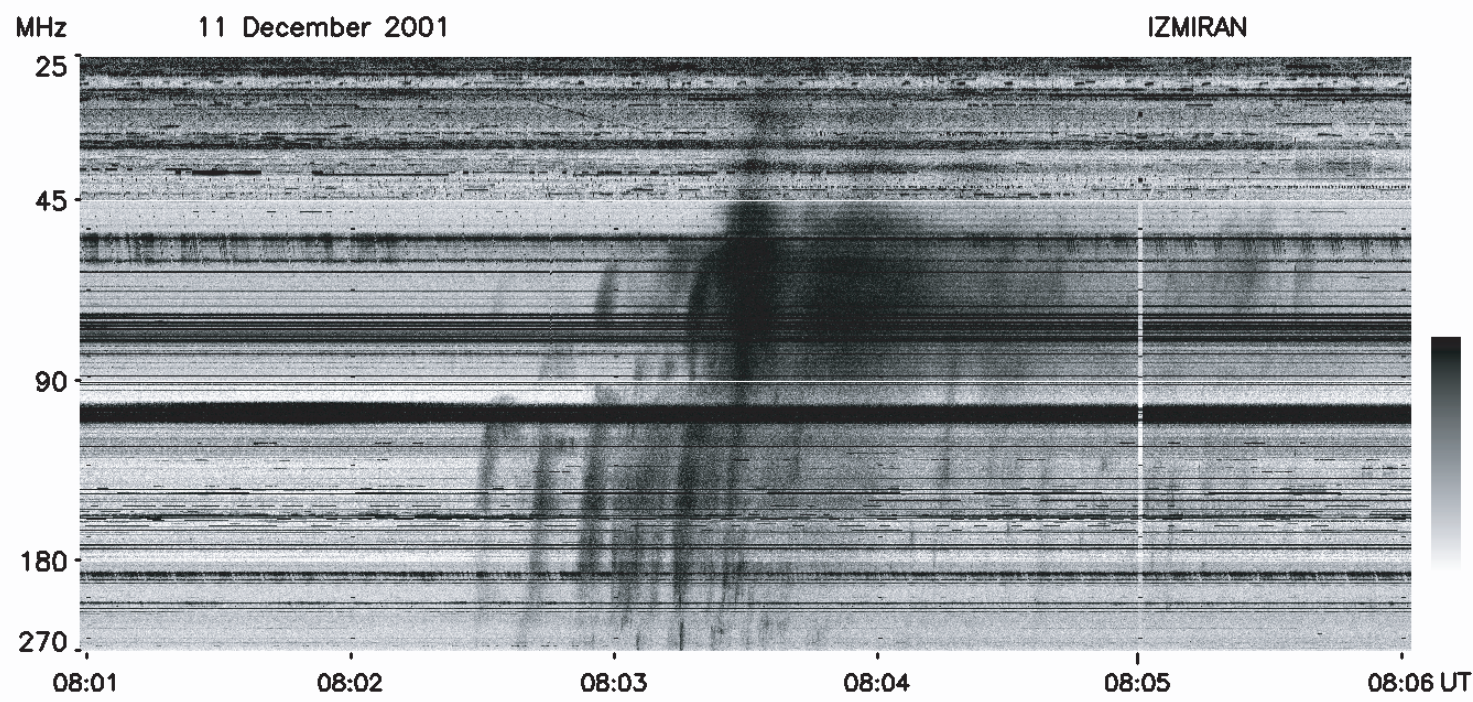

Fig. 3. Dynamic spectrum of the radio emission from the solar corona obtained with IZMIRAN radiospectrograph on December 11, 2001 in the time interval 08:01-08:06 UT. The parallel drifting bands of burst emission starting from about 08:02:30 UT and lasting for about a minute constitute a type III burst group. The intense emission following the type III event is a type V burst.

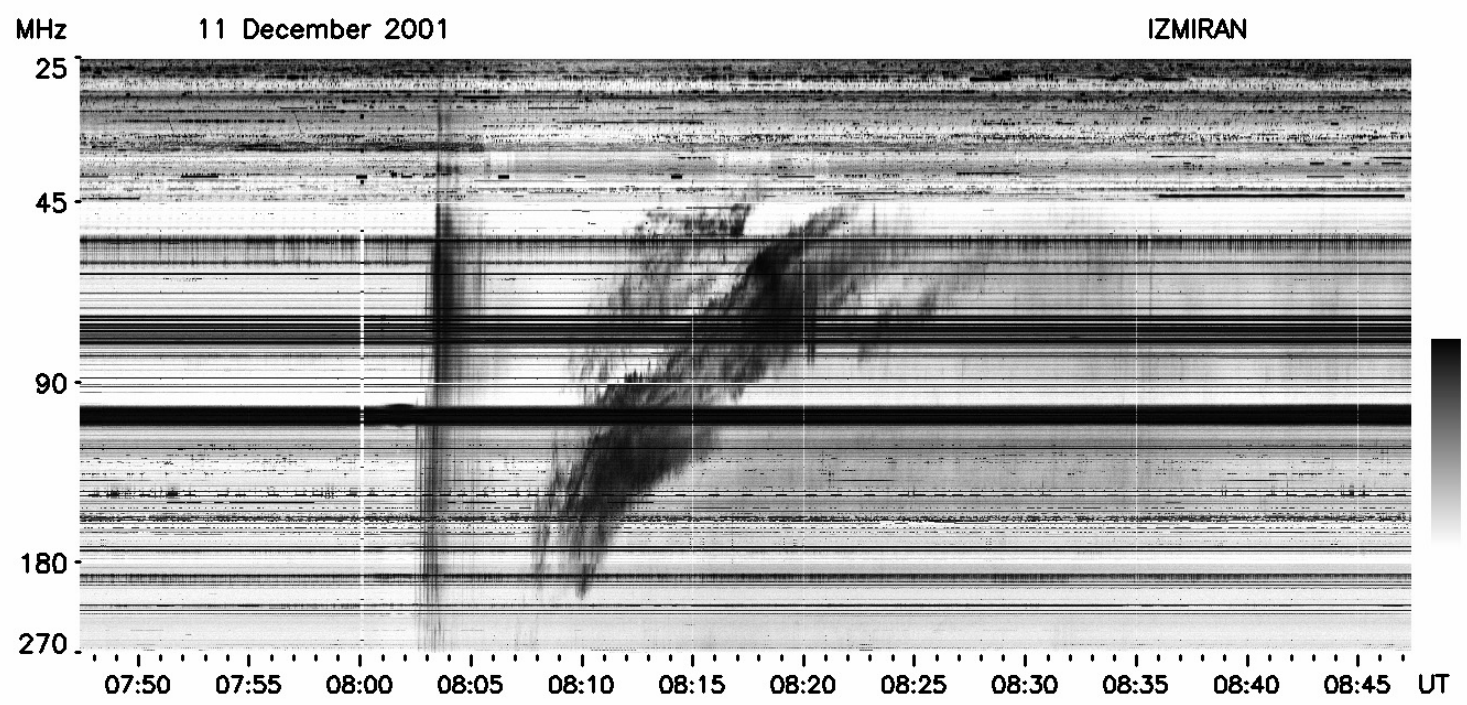

Fig. 4. Same as Fig. 3, but for the time interval 07:45-08:50 UT. All the 3 types of burst (i.e. type II, III and V) mentioned in the text can be noticed in this dynamic spectrum. The type II burst was observed in the frequency range of $\approx 192-46 \mathrm{MHz}$. It started at about 08:08 UT and was first noticed at $46 \mathrm{MHz}$ around 08:13:30 UT.

reasons for the above behaviour is the modulation of the electron beam acceleration/injection process responsible for the type III emission by quasi-periodic oscillations setup by propagating MHD waves in the corona (Aschwanden et al. 1994). Recently, Klassen et al. (1999) showed that groups of fast drift bursts or pulsations with a restricted bandwidth are observed in coronal loops during the impulsive phase of a flare, prior to the onset of type II emission. They addressed them as tracers of a propagating disturbance which later becomes the exciter of type II burst emission. According to them, the disturbance must be a MHD wave or plasma propelled in the ambient corona. The observations of a metric type II burst following the type III emission in the present case strengthens the above argument.
The appearance of parallel drifting bands of emission in the dynamic spectrum in Fig. 3 around the same time as the observed quasi-periodic emission in the single frequency records in Fig. 1 could be considered as an evidence for the presence of propagating MHD waves (Aschwanden et al. 2004). The total duration of the observed emission ( 85 and $97 \mathrm{~s}$, respectively at 151.5 and $109 \mathrm{MHz}$ ), the number of observed pulses (7 and 8) and their period (11.9 and 11.4 s) are in the range of corresponding values reported in the statistical study of type III group emission by Aschwanden et al. (1994). In addition to the above, the observed periodicity is consistent with the characteristic time scale of MHD oscillations set up by a travelling disturbance through the solar corona (Aschwanden et al. 1999). 
Therefore one can calculate the Alfvén speed using the following relation (Roberts et al. 1984),

$p=2.6 \frac{a}{v_{\mathrm{A}}} \mathrm{s}$

where $p$ is the period of the quasi-periodic emission and $a$ is the width of the electron acceleration/injection region. Based on the results of Aschwanden et al. (1994), we assumed $a=$ $3500 \mathrm{~km}$ in the present case. We calculated $v_{\mathrm{A}}$ for the time interval between all the adjacent bursts in Fig. 1, and the mean value is $837 \pm 372 \mathrm{~km} \mathrm{~s}^{-1}(151.5 \mathrm{MHz})$ and $909 \pm 482 \mathrm{~km} \mathrm{~s}^{-1}$ (109 MHz). Note that though the radio emission at 151.5 and $109 \mathrm{MHz}$ originate from different altitude levels in the solar atmosphere, the period and the associated Alfvén speed corresponding to the above two frequencies agree closely. This indicates that the observed quasi-periodicity corresponds most likely to the basic injection period at the acceleration site and is not due to local conditions along the path of the type III electron beams, as pointed out by Aschwanden et al. (1994). The derived value of $v_{\mathrm{A}}$ is also typical of the same region since we have used $a=3500 \mathrm{~km}$, the size of the electron acceleration/injection region reported in the literature. It is interesting to note that $v_{\mathrm{A}}$ derived above is comparable to the drift speed of the coronal type II burst observed on that day following the quasi-periodic type III emission under discussion. Taking clue from the above, we postulate the following scenario for the present observations: the MHD disturbance generated in the aftermath of the $\mathrm{H} \alpha$ flare had first modulated the type III burst acceleration/injection process through oscillations setup in/near the corresponding region. Later it had developed into a shock responsible for the type II bursts observed following the quasi-periodic type III emission. We verified this as follows:

(1) As mentioned earlier, the type III emission reported was first observed at 08:02:23 UT, i.e. about $23 \mathrm{~s}$ from the onset of the associated $\mathrm{H} \alpha$ flare. This implies that the MHD disturbance generated in the aftermath of the latter should have travelled a distance of $22800 \mathrm{~km}\left(\approx 0.03 R_{\odot}\right)$ in the above time interval in order to modulate the type III acceleration/injection process.

(2) The time difference between the onset of the type II and type III emission is $\approx 337 \mathrm{~s}$ (08:08:00-08:02:23 UT). Therefore the MHD disturbance should have travelled a further distance of $\approx 0.48 R_{\odot}$ in the above time interval.

Now, if the same disturbance had been responsible for the observed type II emission also, then the altitude of the plasma level corresponding to the start frequency of the type II emission should be close to the sum of the above two distance estimates, i.e. $\approx 0.51 R_{\odot}$. As pointed out earlier, the type II event was first observed around $192 \mathrm{MHz}$ at 08:08 UT. If we assume that it is due to harmonic emission as reported in the Solar Geophysical Data (February 2002), then a starting frequency of $192 \mathrm{MHz}$ means that the associated coronal shock should have formed at a height where the local plasma frequency was $96 \mathrm{MHz}$. We estimated the altitude of the corresponding plasma level using a $10 \times$ Baumbach-Allen model for the electron density distribution as earlier, i.e.,

$N_{\mathrm{e}}(r)=10 \times 10^{8}\left[\frac{2.99}{r^{16}}+\frac{1.55}{r^{6}}+\frac{0.036}{r^{1.5}}\right] \approx \frac{4 \times 10^{9}}{r^{9}}$

Here $r$ is the radial distance from the center of the Sun and the approximation on the right hand side holds for $1 R_{\odot}<r<$ $1.5 R_{\odot}$. Upon substituting $N_{\mathrm{e}}=1.14 \times 10^{8} \mathrm{~cm}^{-3}$ (the plasma density corresponding to $96 \mathrm{MHz}$ ), we get $r_{96 \mathrm{MHz}} \approx 1.48 R_{\odot}$. The two estimates agree closely with each other. We specifically used Baumbach-Allen model for all the calculations since it holds good upto $r \leq 1.1 R_{\odot}$. Also the recent measurements of electron density in the low corona using YOHKOH soft Xray data by Aschwanden \& Acton (2001) is consistent with the values given by the model. Item (1) above indicates that the type III burst emission in the present case started probably from an altitude of $\approx 1.03 R_{\odot}$, from the center of the Sun. We inferred the electron density at the above location using $10 \times$ BaumbachAllen model, and is $\approx 3.07 \times 10^{9} \mathrm{~cm}^{-3}$. Having known $v_{\mathrm{A}}$ and $N_{\mathrm{e}}$, we calculated the the magnetic field $(B)$ of the region from the definition of the former, i.e.,

$v_{\mathrm{A}}=2.18 \times 10^{6} B N_{\mathrm{e}}^{-1 / 2} \mathrm{~km} \mathrm{~s}^{-1}$

and the value is $\approx 22 \pm 11 \mathrm{G}$.

\section{Conclusions}

We estimated the plasma parameters at the acceleration/injection region of the electron beams responsible for the type III radio burst group observed at 151.5 and $109 \mathrm{MHz}$ on December 11, 2001 around 08:00 UT by assuming that the quasi-periodic nature of the emission is due to oscillations set-up in/near the former by a MHD disturbance propagating through the solar atmosphere in the aftermath of the associated $\mathrm{H} \alpha$ flare. Our findings are:

1) the Alfvén speed in the region is $873 \pm 427 \mathrm{~km} \mathrm{~s}^{-1}$;

2) the electron density in the region is $\approx 3.07 \times 10^{9} \mathrm{~cm}^{-3}$;

3 ) the associated magnetic field is $22 \pm 11 \mathrm{G}$.

Note that the above results should be treated with caution since the solar corona is highly inhomogeneous and we were not able to estimate the plasma parameters, notably $v_{\mathrm{A}}$, with lesser uncertainity. Again, we do not know the start time/frequency of the type III emission reported. It would have helped to obtain the location, electron density and magnetic field of the electron acceleration/injection region more accurately. The $\mathrm{H} \alpha$ flare start time reported is also known only to within $\pm 1 \mathrm{~min}$. It is suggested that further observations of this type with imaging instruments of higher spatial resolution like the Giant Metrewave Radio Telescope (GMRT, Swarup et al. 1991) might provide valuable information on the modulation of acceleration/injection of non-thermal electrons released during a flare (Asai et al. 2001).

Acknowledgements. We thank the staff of the Gauribidnaur radio observatory, University of Mauritius and the Mauritius radio telescope team for their help in the maintenance of the antenna and receiver 
systems. G. N. Rajasekara and C. Nanje Gowda are acknowledged for their assistance in obtaining the MRT and GRH data reported. V. V. Fomichev is thanked for kindly providing the IZMIRAN radio spectrograph data. We benefited from discussions with Y. Sakamato san (NAOJ, Mitaka, Japan) and M. H. Gokhale. We are grateful to the referee for his/her comments on an earlier version of the manuscript that helped us to clearly bring out the results.

\section{References}

Allen, C. W. 1947, MNRAS, 107, 426

Asai, A., Shimojo, M., Isobe, H., et al. 2001, ApJ, 562, L103

Aschwanden, M. J., Benz, A. O., \& Montello, M. L. 1994, ApJ, 431, 432

Aschwanden, M. J., Fletcher, L., Schrijver, C. J., \& Alexander, D. 1999, ApJ, 520, 880

Aschwanden, M. J., \& Acton, L.W. 2001, ApJ, 550, 475

Aschwanden, M. J. 2002, Space Sci. Rev., 101, 178

Aschwanden, M. J., Nakariakov, V. M., \& Melnikov, V. F. 2004, ApJ, 600,458

Baumbach, S. 1937, Astron. Nachrichten, 263, 120

Golap, K., Udaya Shankar, N., Sachdev, S., Dodson, R., \& Sastry, Ch. V. 1998, A\&A, 19, 35

Janssens, T. J., White, K. P., \& Broussard III, R. M. 1973, Sol. Phys., 31,207
Klassen, A., Aurass, H., Klein, K.-L., Hofmann, A., \& Mann, G. 1999, A\&A, 343, 287

Lin, R. P. 1974, Space Sci. Rev., 16, 189

Mangeney, A., \& Pick, M. 1989, A\&A, 224, 242

Mercier, C. 1975, Sol. Phys., 45, 169

Pick, M., \& Ji, S. C. 1986, Sol. Phys., 107, 159

Ramesh, R., Subramanian, K. R., Sundara Rajan, M. S., \& Sastry, Ch. V. 1998, Sol. Phys., 181, 439

Ramesh, R., Kathiravan, C., Satya Narayanan, A., \& Ebenezer, E. 2003, A\&A, 400, 753

Roberts, B., Edwin, P. M., \& Benz, A. O. 1984, ApJ, 279, 857

Solar Geophysical Data, 690, Part I, February 2002

Solar Geophysical Data, 694, Part II, June 2002

Swarup, G., Ananthakrishnan, S., Kapahi, V. K., et al. 1991, Current Sci., 60(2), 95

Vlahos, L. 1986, in Energetic phenomenon on the Sun, ed. M. R. Kundu, \& B. Woodgate (NASA Conf. Publ.), 2439, Chap. 2

Wild, J. P. 1950, Aust. J. Sci. Res., Ser. A, 3, 541

Wild, J. P., Sheridan, K. V., \& Trent, G. H. 1959, in IAU-URSI Symp. Radio Astronomy (Stanford University Press), ed. R. N. Bracewell, 176

Wild, J. P., Smerd, S. F., \& Weiss, A. A. 1963, ARA\&A, 1, 291

Zhao, R.-Y., Mangeney, A., \& Pick, M. 1991, A\&A, 241, 183

Zirin, H., \& Lazareff, B. 1975, Sol. Phys., 41, 425 